\title{
ADDING SHORT SPACINGS TO SYNTHESIS MAPS IN THE
} 'SKY'-DOMAIN

\section{U.J. SCHWARZ}

Kapteyn Laboratory, Postbus 9700AV, Groningen

\section{B.P. WAKKER}

Univ. of Illinois, Dept. of Astronomy, 1002 W. Green St, Urbana IL61801

ABSTRACT We discuss critically the methods used to complement synthesis maps with short-spacing information derived from single-dish observations. The conclusion is that from a single synthesis field the correct result cannot be derived, a large area has to be synthesized.

\section{INTRODUCTION}

For some synthesis observations a lack of short-spacing information can not be tolerated, e.g. if the emission fills the whole primary beam. Galactic hydrogen, for example, has structure at all scales, ranging from a fraction of an arcminute to dozens of degrees. Restoration techniques using only the interferometer data are able to recover short-spacing information corresponding to structures which are smaller than roughly $\Theta_{m}$ in at least one direction, where $\Theta_{m}=\frac{\lambda}{d_{m}-D / 2}$. Here $d_{m}$ is equal to the shortest spacing and $D$ is the diameter of a single element of the interferometer. In order to detect structures larger than $\Theta_{m}$ in all directions, other observations, e.g. from single dishes, are needed. It will be shown however, that single-dish data are insufficient to solve the problem correctly. This is true both for the method described here (which has the advantage of simplicity) and for the conventional approach, in which the short spacings are added in the uv-domain, using the results from a single-dish instrument with size equal to or larger than the interferometer elements.

Below we first discuss a problem with the conventional method, then we describe our alternative. Next follows a discussion of how accurately both methods can recover the short-spacing information.

\section{SAMPLING}

As is well-known, the uv-plane has to be sampled in increments of at least $\mathrm{D} / 2$. In practice, however, this requirement can be relaxed considerably. 


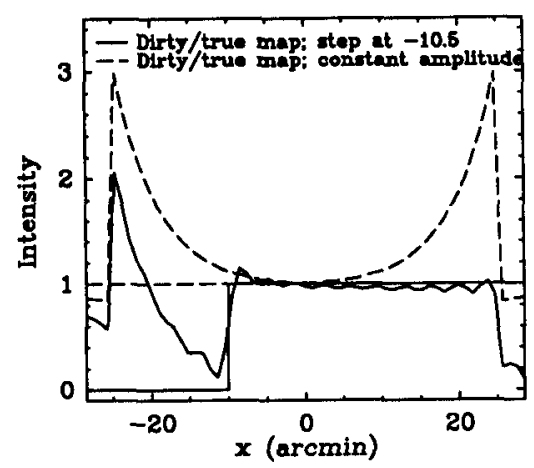

Fig. 1. True distribution and observed distribution as seen with 2/3D sampling for a true distribution with is flat and for one which has a step.

In general it is possible to recover the missing information easily with restoration techniques such as CLEAN; flux distributions which are confined to the unobserved parts of the uv plane are unlikely to be present in astronomical objects. The exception to this is structure that is extended in all directions; such structure is not unlikely to occur, and manifests itself in the short spacings only. So, for the short spacings one needs to be careful to fulfill the sampling theorem. As an example, Fig. 1 shows how large differences between the observed and the true distribution can occur, even if one uses a regular grid with $2 / 3 D$ sampling; this is standard at the WSRT. Although the zero and $2 /{ }_{3} D$ spacings are included this does not give the proper result. Filling in the required additional information necessarily leads to non-regular sampling, giving rise to unwanted responses in the synthesized beam. In order to overcome this, we describe below a method that allows to combine fully sampled single-dish maps with maps in which the missing information at larger spacings was recovered using a deconvolution algorithm (CLEAN, MEM, ...).

\section{DESCRIPTION OF THE METHOD}

In the following we denote functions in the sky domain by lower case letters, and functions in the Fourier (uv-) domain by capital letters.

In interferometric measurements one observes $T \star P$, where $T$ is the visibility of the true brightness distribution $t$, and $P$ is the taper function of an element of the interferometer ( $=$ Fourier transform of the primary beam $p$ ). A single dish produces a map equal to

$$
t_{\mathrm{ad}}=t \star p_{\mathrm{sd}}
$$

Usually one deduces the missing short spacings from the single-dish observations directly; this requires a deconvolution of the single-dish map. Such a deconvolution is not without problems: (i) large-scale structures influence the map due to far side-lobes; (ii) systematic errors can be present 

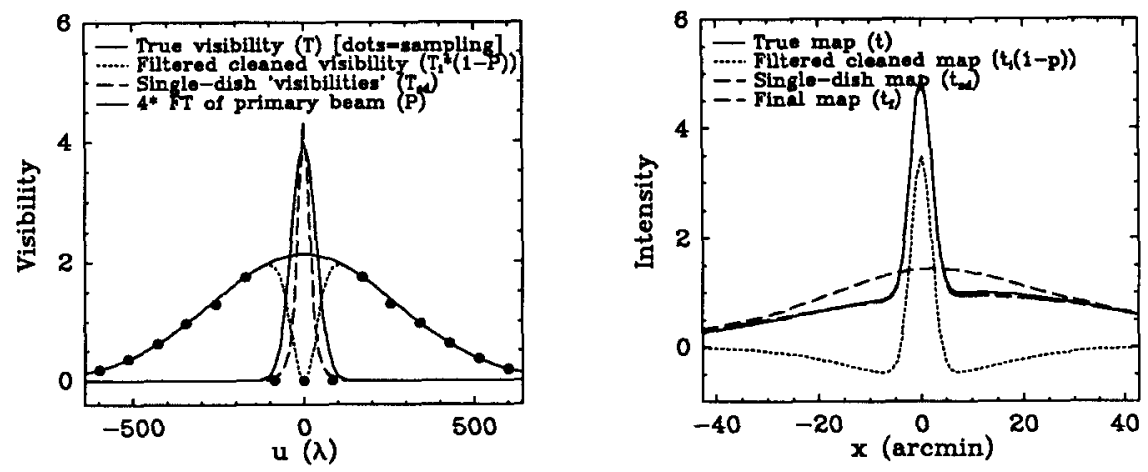

Fig. 2. Visibilities and intensities for the model distribution and for the interferometer and single-dish observations.

(varying baselines, etc); (iii) the visibility $T$ generally increases rapidly toward short spacings (see example in Fig. 2); (iv) the taper function $P$ drops rapidly at spacings around $\mathrm{D} / 2$ (Fig. 2); (v) the observed field of the single-dish observations is often small, resulting in edge effects.

These problems make the deconvolution rather uncertain and can lead to the amplification of errors. Therefore we think it is more reliable to use the single-dish map as it is and to "add" the synthesis map to it.

We use $t_{\mathrm{i}}$ for the synthesis map after "cleaning" (or another restoration) and after correction for the primary beam attenuation. $t_{\mathrm{sd}}$ is the single-dish map. Then we construct the final map $t_{\mathrm{f}}$ as:

$$
t_{\mathrm{f}}=(1-p) \star t_{\mathrm{i}}+t_{\mathrm{sd}}
$$

The term $p \star t_{\mathrm{i}}$ is the convolution of the synthesis map with the primary beam. This term must be subtracted, because $t_{\text {sd }}$ already contains this contribution. In the Fourier domain (see Fig. 2) the subtraction corresponds to filtering the low spatial frequencies out of the synthesis map. That means that part of the effect of the deconvolution is thrown away. Only the part where the taper function of the single dish is low is kept. This is especially important if one uses single dishes of the same size as the interferometer elements. One remarks a similarity with the "MultiResolution CLEAN" (MRC, Wakker \& Schwarz 1988); the method described above can in fact be considered as the final step of MRC.

The advantages are: (i) no deconvolution of the single dish map is needed; (ii) one sees directly what it does; (iii) scaling is no problem; (iv) the problem of irregular sampling is avoided. 

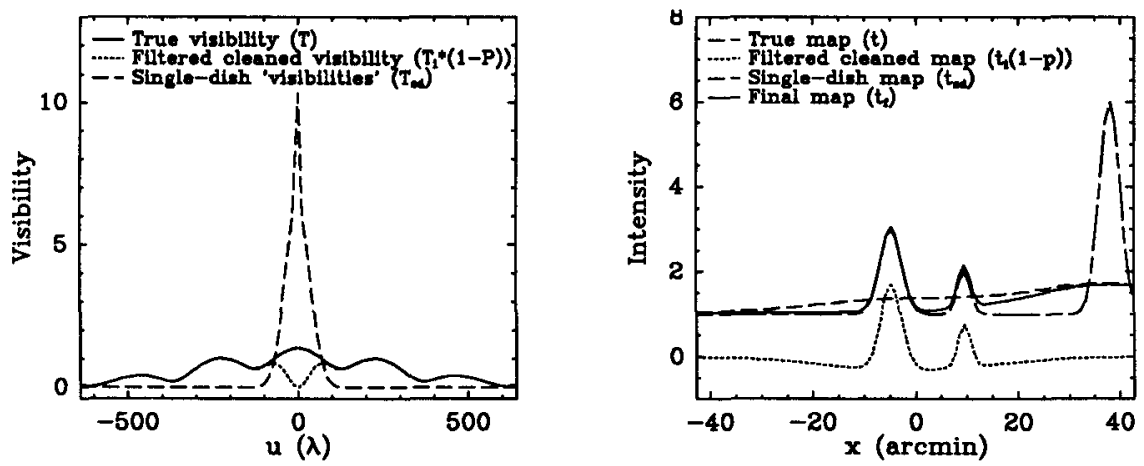

Fig. 3. An example showing how a distant point-source can lead to spurious flux in the final map when combining interferometer and single-dish data.

\section{CRITICAL EXAMINATION OF THE METHOD}

In order to understand more fully what the addition of the shortspacing information really does, we write $t$ as $t_{\mathrm{s}}+t_{\mathrm{e}}$; with $t_{\mathrm{s}}$ the small-scale structure and $t_{\mathrm{e}}$ the extended structure. From the synthesis map one finds $t_{\mathrm{i}}=t_{\mathrm{s}}$ and the single-dish gives $t_{\mathrm{sd}}=t_{\mathrm{B}} \star p+t_{\mathrm{e}} \star p$. Inserting this into Eq. 2 results in

$$
t_{\mathrm{f}}=t_{\mathrm{s}}+t_{\mathrm{e}} \star p .
$$

If $t_{\mathrm{e}}$ is really very extended, then $t_{\mathrm{e}} \star p$ is approximately equal to $t_{\mathrm{e}}$ and we have the desired result $t_{\mathrm{f}}=t_{\mathrm{s}}+t_{\mathrm{e}}=t$.

A complication is, however, that $t_{\mathrm{s}}$ is most likely not restricted to the region of the observation. We therefore write

$$
t_{\mathrm{s}}=t_{\mathrm{so}}+t_{\mathrm{sx}}
$$

where $t_{\mathrm{so}}$ is the small-scale structure inside the field of view of the synthesis map and $t_{\mathrm{sx}}$ is the fine-structure outside the field. Now $t_{\mathrm{i}}=t_{\mathrm{so}}$ and $t_{\mathrm{sd}}=$ $t_{\mathrm{so}} \star p+t_{\mathrm{sx}} \star p+t_{\mathrm{e}} \star p$. This yields

$$
t_{\mathrm{f}}=t_{\mathrm{s}}+t_{\mathrm{e}}+t_{\mathrm{sx}} \star p .
$$

The last term shows, that the "outside" fine structure can be beamsmeared into the field of view and is not corrected for. Therefore it can alter the final map. The effect is shown schematically in Fig. 3. The same error can arise if one uses the conventional method of extracting the short spacings from the single-dish map. Such small-scale structure can be mistaken for extended emission. The only way to avoid this effect is to synthesize a larger region, in fact "mosaicing" it.

\section{REFERENCES}

Wakker B.P, Schwarz U.J., 1988, Astr. Ap., 200, 312 